\title{
Single-Photon Hologram of a Zero-Area Pulse
}

\author{
Michał Lipka $\oplus^{1, *}$ and Michał Parniak $\oplus^{1,2, \dagger}$ \\ ${ }^{1}$ Centre for Quantum Optical Technologies, Centre of New Technologies, University of Warsaw, Banacha 2c, 02-097 Warsaw, Poland \\ ${ }^{2}$ Niels Bohr Institute, University of Copanhagen, Blegdamsvej 17, 2100 Copenhagen, Denmark
}

(Received 5 May 2021; accepted 17 September 2021; published 12 October 2021)

\begin{abstract}
Single photons exhibit inherently quantum and unintuitive properties such as the Hong-Ou-Mandel effect, demonstrating their bosonic and quantized nature, yet at the same time may correspond to single excitations of spatial or temporal modes with a very complex structure. Those two features are rarely seen together. Here we experimentally demonstrate how the Hong-Ou-Mandel effect can be spectrally resolved and harnessed to characterize a complex temporal mode of a single-photon—a zero-area pulse—obtained via a resonant interaction of a terahertz-bandwidth photon with a narrow gigahertz-wide atomic transition of atomic vapor. The combination of bosonic quantum behavior with bandwidth-mismatched light-atom interaction is of fundamental importance for deeper understanding of both phenomena, as well as their engineering offering applications in characterization of ultrafast transient processes.
\end{abstract}

DOI: $10.1103 /$ PhysRevLett.127.163601

Single photons (SPs) exhibit a plethora of highly nonclassical features manifesting their quantized and bosonic nature and demonstrating the meanders of quantum theory. One of classic examples is the Hong-Ou-Mandel (HOM) effect [1]. When two identical photons enter two respective input ports of a balanced beam splitter (BS), the photons always leave together via a single output port. In consequence, no coincidences between the ports can be observed. This unintuitive feature fundamentally stems from the destructive interference of two-photon amplitudes corresponding to two scenarios each with one of the photons reflected and one transmitted. Interestingly, observing reminiscent coincidences with a single-photon camera (i.e., spatially and angularly resolved) allows one to probe and localize the wave front differences of the two photons, which has been leveraged to precisely measure a single-photon wave front in a method reminiscent of classical holography [2]. Two-photon interferograms, measured with either spatial-angular or temporal-spectral resolution, are also at the core of superresolution imaging [3], quantum fingerprinting [4-6], and photon-pair source characterization [7-10]. The spectrally resolved HOM effect has been measured with a dispersive fiber spectrometer $[11,12]$. HOM interference extends beyond the photonic realm and has been shown for other bosonic (quasi) particles, such as atoms $[13,14]$, phonons [15], or spin waves $[16,17]$.

Published by the American Physical Society under the terms of the Creative Commons Attribution 4.0 International license. Further distribution of this work must maintain attribution to the author(s) and the published article's title, journal citation, and DOI.
The nonclassical features of SPs become intriguing when the spatial or temporal mode has a nontrivial structure with added qualitative features, such as orbital angular momentum (OAM). In the spatial domain, mode structuring has led to insights and applications, such as remote object identification [18], improved sensitivity [19], and uncertainty relations for OAM [20]. However, with mostly single-mode optical architectures, the mode engineering in time-frequency (TF) degrees of freedom (d.o.f.) attracts most attention, enabling bandwidth matching [21], generating and manipulating high-dimensional entanglement on chip [22], and studies of global versus local two-photon interference (TPI) in quantum networks [23].

SPs exhibit complex light-matter interactions, mostly studied in the classical regime and leading to complex modal structure. One such a case is the resonant interaction of an ultrafast pulse with a slowly relaxing medium, e.g., a femtosecond pulse passing through atomic vapor-a case demonstrated to produce zero-area (ZA) or $0 \pi$ pulses $[24,25]$ with temporal envelopes consisting of alternating \pm sign lobes. This interaction has been demonstrated for SP states and Rb vapor [26], exploring a vastly unharnessed region of light-matter interaction between a terahertzbandwidth photon and a gigahertz-wide (Dopplerbroadened) atomic transition. While the photon is rarely absorbed, the interaction is highly dispersive, leaving the photon in the ZA temporal shape and imprinting a spectral phase (SPHI). Costanzo et al. [26] characterized SP ZA pulses in the temporal domain via homodyne detection, which is a robust tomography method, yet never provides optimal information due to inherent shot noise. Singlephoton holography can directly reconstruct the SPHI resulting from bandwidth-mismatched (BM) light-matter interaction, without local oscillator (LO) optimization and 
the shot noise contribution inherent to homodyne techniques. Characterizing the SPHI at a single-photon level is of fundamental importance for quantum coherent control techniques $[27,28]$ and utilization of the TF d.o.f. in quantum networks [23]. High-bandwidth ultrafast photons can also efficiently interact with atomic vapors via two-photon transitions $[27,29]$. Furthermore, ultrafast frequency combs have been used to probe temporal dynamics of two-photon transitions via direct frequency comb spectroscopy [30], combining high temporal resolution and a vast spectral range.

In this Letter, we combine two highly nonintuitive concepts and demonstrate spectral single-photon holography (SSPH) applied to the characterization of ultrafast ZA SP pulses. The idea of our experiment is depicted in Fig. 1. We employ spontaneous parametric down-conversion (SPDC) to produce pairs of SPs - an idler and a signalwith central wavelengths of $795 \mathrm{~nm}$. The signal photon with $10 \mathrm{~nm}$ bandwidth passes through a hot $\mathrm{Rb}$ vapor, resonantly interacting with the D1-line electronic transition, and obtaining both a ZA temporal shape and intrinsically a nontrivial SPHI profile imprinted through light-matter interaction. The photon pair is then interfered on a BS. The output ports $( \pm)$ are analyzed with a single-photon spectrometer and coincidences in wavelength coordinates $\left(\lambda_{ \pm}\right)$are counted. The inset of Fig. 1 depicts a simulated coincidence map. In the absence of the BS and the Rb cell, the coincidence map would just be the joint spectral intensity (JSI) of the twophoton state. Notably, if only the Rb was removed, we would ideally see no coincidences at all, indicating the SPHIs of the two-photons are identical. A coincidence pattern

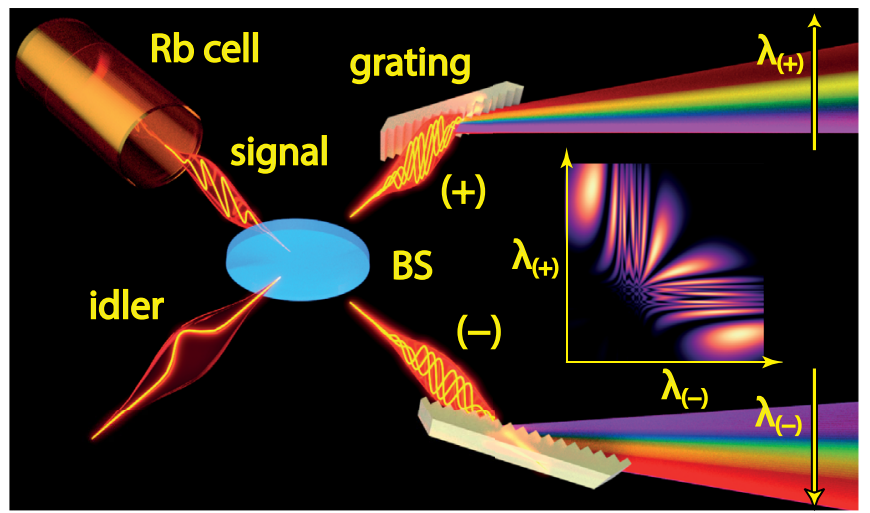

FIG. 1. Spectral single-photon holography of an ultrafast ZA photon. Starting from a pair of identical broadband $(10 \mathrm{~nm}$, $100 \mathrm{fs}$ ) single photons (signal, idler), one (signal) interacts with hot ${ }^{87} \mathrm{Rb}$ vapor, forming a ZA temporal shape and acquiring a spectral phase. Signal and idler photons are interfered on a balanced BS where output \pm modes are spectrally resolved via diffraction gratings. Coincidence detection between wavelength $\lambda_{ \pm}$components shows a footprint of the ZA single photon's SPHI - a manifest of the HOM effect. Inset: simulated map of spectrally resolved $\left(\lambda_{ \pm}\right)$coincidences-an analog of classical interferogram. uniquely corresponds to a SPHI difference between the photons and carries a footprint of the interaction between a terahertz-bandwidth photon and a gigahertz atomic transition.

Fundamentally, our demonstration combines a vastly unexplored regime of bandwidth-mismatched light-matter interaction with a purely quantum effect of TPI. The previous SP ZA pulses demonstration [26] employed temporal homodyne detection requiring $\mathrm{LO}$ temporal mode optimization [31]. In comparison, SSPH does not require LO or any optimization, working readily for any kind of SPHI profiles. Importantly for quantum metrology, our method also avoids the shot noise inherent to homodyning.

We envisage applications of SSPH for characterization of ultrafast transient phenomena such as chemical reactions or for biological measurements (see Supplemental Material [32]), which could benefit from negligible absorption of the probe photons. Ultrafast pulses have proved indispensable where time resolution is required, e.g., for time-resolved photoemission tomography of molecular orbitals [45] and probing the transition states of chemical reactions $[46,47]$ or their coherent control $[48,49]$. SSPH can supplement these methods as recent times see more and more proposals for using quantum light [50,51] and TPI effects in spectroscopy [52]. For instance, femtosecond transition state spectroscopy $[46,47,53]$ relies on a pair of pump and delayed probe femtosecond pulses. SSPH could replace the typical probe fluorescence signal for a direct noninvasive characterization. SP operation also promises flexibility in molecular control [54] via quantum nondemolition continuous measurements [55], and relying on the TPI and coincidence postselection provides robustness to the noise from scattering surroundings of the biological samples. While being noninvasive, the SSPH remains an ultrafast and interferometric scheme able to replace probe signals in different spectroscopic methods [56,57]. The advantage of noninvasive SP probing can be quantified with Fisher information per damage to the sample [58].

Our experimental setup consists of a SP source [Fig. 2(a)] and of a SSPH part [Fig. 2(b)]. To produce ultrashort SP states, we employ the type-I noncollinear SPDC process in a beta barium borate (BBO, $2 \mathrm{~mm}$ length along optical axis), pumped with a focused pump beam with $397.5 \mathrm{~nm}$ central wavelength, Gaussian beam radius of $w_{0}=70 \mu \mathrm{m}$, and $100 \mathrm{~mW}$ average power. To obtain the blue pump, we employ second harmonic generation of femtosecond pulses (100 fs, central wavelength of $795 \mathrm{~nm}$ ) from a Ti:Sapphire laser (Spectra-Physics MaiTai), in a second BBO crystal (0.5 mm length along optical axis). The SPDC emission is spectrally filtered with an interference filter (FWHM $10 \mathrm{~nm}$ ) tilted to have the central transmission wavelength at $796.7 \mathrm{~nm}$. Two Gaussian transverse modes are selected by coupling to single-mode fibers. The modes are chosen for highest correlation and SP brightness. Selected modes, corresponding to signal and 
(a)

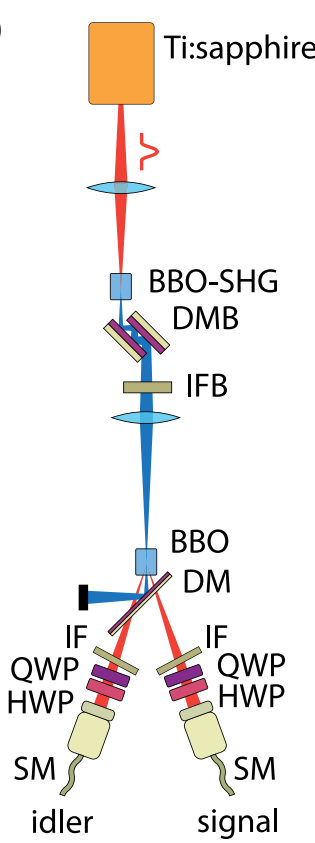

(b)

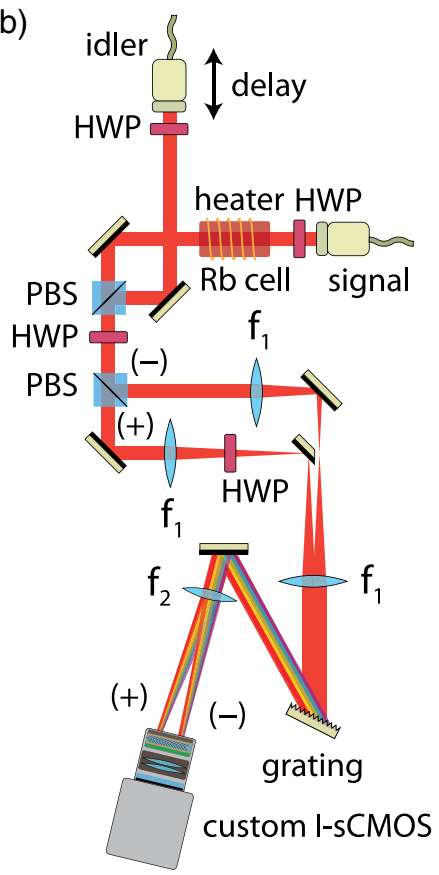

FIG. 2. (a) Source of single-photon pairs. 100 fs pulses from Ti: Sapphire laser (central wavelength $795 \mathrm{~nm}$ ) are frequency doubled in a BBO crystal (BBO-SHG) and used to pump type-I SPDC in the second crystal (BBO). Pairs of photons are spectrally filtered (IF, see main text) and coupled to singlemode polarization-maintaining (PM) fibers (single mode, SM) which select highest-correlated transverse modes from the SPDC emission cone. Dichroic mirrors (DM for $\approx 800 \mathrm{~nm}, \mathrm{DMB}$ for $\approx 400 \mathrm{~nm}$ ) and a $400 \mathrm{~nm}$ bandpass (40 nm FWHM) interference filter (IFB-IF for $400 \mathrm{~nm}$ ) separate pumping beams. Quarter- and half-wave plates (QWPs and HWPs, respectively) allow for polarization matching to the PM fiber. (b) Setup for SSPH. Signal photon passes through heated $\mathrm{Rb}$ cell, interacting resonantly with $\mathrm{D} 1 \mathrm{Rb}$ line and obtaining a SPHI profile. The output \pm modes of the interferometer are spatially separated on another PBS. Imaging setup (focal length $f_{1}=150 \mathrm{~mm}$ ) superimposes the \pm modes spatially on a diffraction grating while separating them angularly. The last HWP in the $(+)$ mode path rotates the polarization to be perpendicular to the grating grooves, ensuring maximal efficiency. The grating is far-field imaged (focal length $f_{2}=300 \mathrm{~mm}$ ) onto an intensified single-photon-sensitive camera (I-sCMOS), spatially separating the spectral components of \pm modes into distinct camera frame regions $( \pm)$.

idler photons, enter the second part of the setup. Signal photons pass through a heated $\mathrm{Rb}$ vapor cell. The cell temperature controls the optical depth (OD) (see Supplemental Material [32]), which determines the strength of light-matter coupling. The photon leaves the $\mathrm{Rb}$ cell in a ZA temporal shape and with its SPHI given by a single Lorentzian resonance profile centered on the $\lambda_{0}=795 \mathrm{~nm}$ D1 Rb line [34],

$$
\varphi_{s}(\lambda)=\mathrm{OD} \times \frac{x(\lambda)}{1+x(\lambda)^{2}}
$$

where $x(\lambda)=2 \pi \tau c\left(\lambda-\lambda_{0}\right) / \lambda_{0}^{2}$, with $c$ denoting the speed of light and where $\tau$ is the Doppler-broadened lifetime of the excited state ranging from 215 to $240 \mathrm{ps}$ for employed $\mathrm{Rb}$ temperatures (see Supplemental Material). The idler photon is delayed to match the signal (the linear component of the SPHI is compensated). The photon pair is then interfered on a polarization-based equivalent of a balanced BS, a setup employed previously in Ref. [2]. Initially, the signal and idler photons have their polarizations rotated to vertical and horizontal, respectively, which allows superimposing their spatial modes on a polarizing beam splitter (PBS). The photons' polarizations are then jointly rotated on a half-wave plate, to diagonal and antidiagonal, respectively. Finally, the photons interfere and are spatially separated by another PBS for which the output ports correspond to the \pm outputs and are far-field imaged onto a mirror and a D-shaped mirror, respectively, allowing one to separate the ports angularly, while imaging both on a single diffraction grating (1200 lines/mm, $750 \mathrm{~nm}$ blaze). The grating is far-field imaged on an ultrafast intensified CMOS camera $[59,60]$. On the camera frame, the \pm ports appear as $140 \times 5$ pixel regions, with the longer dimension corresponding to $\lambda_{ \pm}$. We collect $8.2 \times 10^{4}$ camera frames per second with an average of $\bar{n} \approx 0.2$ photons per frame $\left(1.4 \times 10^{-4}\right.$ per pixel). The camera pixels are not photonnumber resolving; however, on average, we expect two or more photons to be misclassified as a SP only once per $7.2 \times 10^{4}$ frames (see Supplemental Material [32]).

Let us denote by $0 \leq n\left(\lambda_{ \pm}\right) \leq 5$ the number of photons registered at the $\lambda_{ \pm}$coordinates in a single frame (one photon per pixel, 5 pixels per spectral point) and by $\langle$.$\rangle the$ average over collected frames. A raw coincidence map $\mathcal{R}\left(\lambda_{+}, \lambda_{-}\right)=\left\langle n\left(\lambda_{+}\right) n\left(\lambda_{-}\right)\right\rangle$is a normalized histogram of events where a photon pair is registered in a single frame with the first (second) photon in the $+(-)$ region at the $\lambda_{+}$ $\left(\lambda_{-}\right)$coordinate. We subtract accidental coincidences $\mathcal{A}\left(\lambda_{+}, \lambda_{-}\right)=\left\langle n\left(\lambda_{+}\right)\right\rangle\left\langle n\left(\lambda_{-}\right)\right\rangle$to obtain the coincidence map

$$
\mathcal{C}\left(\lambda_{+}, \lambda_{-}\right)=\mathcal{R}\left(\lambda_{+}, \lambda_{-}\right)-\mathcal{A}\left(\lambda_{+}, \lambda_{-}\right),
$$

which is the photon-number covariance. The subtraction is required due to many experiment repetitions per single camera frame, creating artificial coincidences (see Supplemental Material [32]). To predict the form of $\mathcal{C}\left(\lambda_{+}, \lambda_{-}\right)$we consider the two-photon component of the signal $(s)$ and idler $(i)$ joint wave function $\Psi\left(\lambda_{s}, \lambda_{i}\right)$. The probability of observing a coincidence at spectral coordinates $\lambda_{ \pm}$of $\pm \mathrm{BS}$ ports is given by

$$
P_{c}\left(\lambda_{+}, \lambda_{-}\right)=\frac{1}{4}\left|\Psi\left(\lambda_{+}, \lambda_{-}\right)-\Psi\left(\lambda_{-}, \lambda_{+}\right)\right|^{2} .
$$

We assume that the photons are identical except for the SPHI $\varphi_{s}(\lambda)$ of the signal mode, i.e., $\Psi\left(\lambda_{-}, \lambda_{+}\right)=$ $\Psi\left(\lambda_{+}, \lambda_{-}\right) \exp \left\{i\left[\varphi_{s}\left(\lambda_{-}\right)-\varphi_{s}\left(\lambda_{+}\right)\right]\right\}$. Hence, 
$P_{c}\left(\lambda_{+}, \lambda_{-}\right)=\frac{1}{2}\left\{1-\cos \left[\varphi_{s}\left(\lambda_{+}\right)-\varphi_{s}\left(\lambda_{-}\right)\right]\right\} \times\left|\Psi\left(\lambda_{+}, \lambda_{-}\right)\right|^{2}$,

where the first term lets us reconstruct the SPHI $\varphi_{s}(\lambda)$ and the second corresponds to the JSI of the photon pair. Additional mode mismatch between the photons decreases the interference visibility, corresponding to $\cos (\ldots) \rightarrow$ $\mathcal{V} \cos (\ldots)$ for visibility $\mathcal{V} \leq 1$.

The coincidence maps for three $\mathrm{Rb}$ cell temperatures $\left(T_{1}=188^{\circ} \mathrm{C}, T_{2}=174{ }^{\circ} \mathrm{C}, T_{3}=86^{\circ} \mathrm{C}\right.$,) are presented in Fig. 3 along with theoretical predictions. The fidelity $0 \leq$ $\mathcal{F} \leq 1$ [61] between the experimental and theoretical maps normalized to a unit sum, yields $94 \%, 86 \%$, and $89 \%$ for $T_{1}, T_{2}$, and $T_{3}$, respectively. In all maps the coincidences are most strongly present on a broad stripe along the antidiagonal. This feature stems from JSI $\left|\Psi\left(\lambda_{+}, \lambda_{-}\right)\right|^{2}$ of the SPDC-photon pairs, which in our case are spectrally correlated. See Supplemental Material [32] for comparison of simulated interferograms with correlated and uncorrelated photons and JSI maps. The characteristic cross at $\lambda_{ \pm}=795 \mathrm{~nm}$ corresponds to the $\mathrm{Rb}$ resonance where the phase variation becomes too rapid to be resolved. The coincidences in this region correspond to a phase-averaged case. The phase sign flip around $\lambda_{0}$, illustrated in the right column of Fig. 3, follows from Eq. (1). While the brief interaction between a SP and $\mathrm{Rb}$ atoms has the most pronounced spectral footprint at higher temperatures, corresponding to fitted optical depths of $\mathrm{OD}\left(T_{1}\right)=4.6 \times$ $10^{3}$ and $\operatorname{OD}\left(T_{2}\right)=2.6 \times 10^{3}$, the presence of much cooler $\mathrm{Rb}$ at $T_{3}$ is still distinctly identifiable, despite a comparably low $\mathrm{OD}\left(T_{3}\right) \approx 20$ yielding a peak-to-peak SPHI variation of $20 \mathrm{rad}$. This observation suggests metrological applications in nondisturbing sensing of the sample presence. The OD values corresponding to SPHIs reconstructed from the experimental data are in good agreement with the theory prediction for independently measured $\mathrm{Rb}$ cell temperatures. Even without prior knowledge of the phase profile $\varphi(\lambda)$, standard holographic reconstruction techniques [36,37] can extract $\varphi(\lambda)$ since the problem is analogous to the processing of classical interferograms. We have verified the Fourier-domain retrieval of $\varphi_{s}\left(\lambda_{+}\right)-\varphi_{s}\left(\lambda_{-}\right)$ modulo $2 \pi$, for the case of $T_{1}=188^{\circ} \mathrm{C}$, (see Supplemental Material [32]).

The interference visibility in SSPH is not only a benchmark of the setup quality, but estimated locally $\mathcal{V}\left(\lambda_{+}, \lambda_{-}\right)$ carries unique information. The total phase $\varphi_{s}\left(\lambda_{+}\right)-$ $\varphi_{s}\left(\lambda_{-}\right)$estimated from the coincidence map is a twodimensional abundant representation of a one-dimensional $\varphi_{s}(\lambda)$ enhancing estimation of $\varphi_{s}(\lambda)$ and guaranteeing that the cos term in Eq. (4) cannot be constant over the spectral range of $\varphi_{s}(\lambda)$ unless equal to unity (in which case $P_{c}=0$ ). Hence, when the local visibility $\mathcal{V}\left(\lambda_{+}, \lambda_{-}\right)$falls to zero over

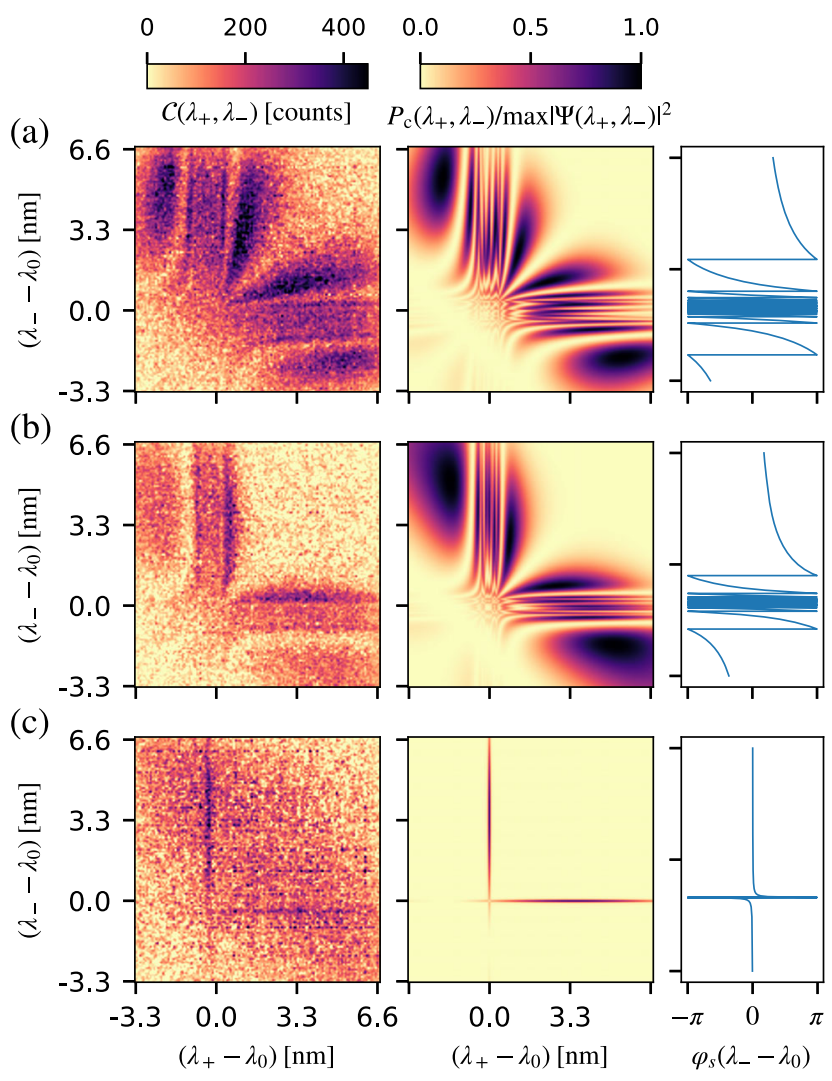

FIG. 3. Spectral single-photon holograms of an ultrafast $100 \mathrm{fs}$ photon resonantly interacting with $\mathrm{Rb}$ vapor heated to (a) $T_{1}=188{ }^{\circ} \mathrm{C}$, (b) $T_{2}=174{ }^{\circ} \mathrm{C}$, (c) $T_{3}=86{ }^{\circ} \mathrm{C}$. Left column: experimental results, i.e., the observed photon-number covariance $\mathcal{C}\left(\lambda_{+}, \lambda_{-}\right)$(coincidences with subtracted background) in spectral coordinates $\lambda_{ \pm}-\lambda_{0}$ between \pm ports of a BS. The BS interferes the measured photon with the reference. Central column: corresponds to a theoretical prediction of a spectrally resolved coincidence probability $P_{c}\left(\lambda_{+}, \lambda_{-}\right)$with no imperfections $(\mathcal{V}=1)$, as given by Eq. (4). Right column: theoretical prediction for the SPHI $\varphi_{s}\left(\lambda_{-}-\lambda_{0}\right)$ modulo $2 \pi$, given by Eq. (1).

extended regions with coincidences present $P_{c}\left(\lambda_{+}\right.$, $\left.\lambda_{-}\right) \neq 0$, it is a strong indicator of rapid oscillations below resolution (averaging cos to zero). The method can thus detect presence of spectral features on subresolution scales. Finally, we have quantified the visibility by dividing a smoothened version of the experimental coincidence map into square regions (1 $\mathrm{nm}$ side length) and locally estimating the visibility from the maximal and minimal value within the region. This way we obtained average visibility of $\mathcal{V} \approx 0.69 \pm 0.16, \mathcal{V} \approx 0.79 \pm 0.12$, and $\mathcal{V} \approx 0.88 \pm 0.09$ for $T_{1}=188^{\circ} \mathrm{C}, T_{2}=174^{\circ} \mathrm{C}$, and $T_{3}=86^{\circ} \mathrm{C}$, respectively, where the uncertainties correspond to 1 standard deviation across the regions. For more details, see the Supplemental Material [32]. For comparison, using classical light the maximal attainable visibility of TPI is $50 \%$.

Metrological advantage of SSPH over homodyne detection.-In conventional homodyning, we measure a 
variance of homodyne current of the signal SP. To gain full information, we consider a multipixel measurement and analyze covariance (see Supplemental Material for setup examples [32]), which has been previously considered in the time domain [62]. The homodyne signal is zero mean with covariance of $\sim\left\{\frac{1}{2} \delta\left(\lambda_{+}-\lambda_{-}\right)+\left|\psi_{s}\left(\lambda_{+}\right) \psi_{s}\left(\lambda_{-}\right)\right| \times\right.$ $\left.\cos \left[\varphi_{s}\left(\lambda_{+}\right)-\varphi_{s}\left(\lambda_{-}\right)\right]\right\}$with an inherent shot noise component (first term) and $\psi_{s}(\lambda)$ being the SP wave function, which is in contrast to SSPH [Eq. (4)]. To further elucidate the advantage, consider a more direct scenario of selfguided tomography [63], where spectrotemporal shaping is employed both for a LO and the reference SP. We strive to shape the reference to be the same as the signal photon. The homodyne case involves maximizing the inherently noisy variance. The SSPH involves minimization of the coincidence count, which becomes a low-noise signal with no offset. This can be directly demonstrated by considering the estimation of residual distinguishability $\alpha \ll 1$ between signal and reference modes, as derived in the Supplemental Material [32] in the context of Fisher information $F_{\alpha}$ per experimental shot. We obtain $F_{\alpha} \sim \alpha^{-1 / 2}$ for the homodyning and $F_{\alpha} \sim \alpha^{-1}$ for the SSPH, which unequivocally demonstrates the preferable scaling of the SSPH. With no prior knowledge $(\alpha \approx 1)$ a hybrid of homodyning followed by SSPH may be beneficial.

In this Letter, we experimentally combined highly unintuitive phenomena bringing together the bosonic and quantum nature of SP light and the complex spectral structure of ultrafast ZA pulses obtained in resonant BM interaction between a SP and ${ }^{87} \mathrm{Rb}$ vapors. Our experiment demonstrates how the Hong-Ou-Mandel effect can be spectrally resolved and harnessed for characterization of ultrafast SPs in a holographylike method. The broadband ultrafast photons carry a unique spectral footprint of the resonant interaction with narrow atomic transitions, corresponding to their ZA temporal shape. Demonstrated herein, SSPH both supplements and extends previous homodyne measurements of SP ZA pulses, as well as promises unique applications in probing ultrafast transient phenomena such as picosecond-scale chemical reactions. Notably, no matching of the reference to the photon is required. This may, on one hand come, as a surprise, since visibility of interference is reduced as the two photons cease to match in the time domain. Our method solves this via spectrally resolved detection, where local visibility of spectral HOM interference is high. Furthermore, it broadens the fundamental understanding of SP BM light-atom interactions and brings closer the prospect of engineering those phenomena for the range of applications. Apart from spectroscopic applications, such interactions may find natural applications in quantum information processing in the spectral domain [64-66].

This scientific work has been funded by Polish science budget funds for years 2019-2023 as a research project within the "Diamentowy Grant" program of the Ministry of
Education and Science (DI2018 010848), by the Foundation for Polish Science (MAB/2018/4 "Quantum Optical Technologies") and by the Office of Naval Research (N62909-19-1-2127). The "Quantum Optical Technologies" project is carried out within the International Research Agendas program of the Foundation for Polish Science cofinanced by the European Union under the European Regional Development Fund. We would like to thank M. Jachura and W. Wasilewski for fruitful discussions and $\mathrm{K}$. Banaszek for the generous support.

*m.lipka@cent.uw.edu.pl m.parniak@cent.uw.edu.pl

[1] C. K. Hong, Z. Y. Ou, and L. Mandel, Phys. Rev. Lett. 59, 2044 (1987).

[2] R. Chrapkiewicz, M. Jachura, K. Banaszek, and W. Wasilewski, Nat. Photonics 10, 576 (2016).

[3] M. Parniak, S. Borówka, K. Boroszko, W. Wasilewski, K. Banaszek, and R. Demkowicz-Dobrzański, Phys. Rev. Lett. 121, 250503 (2018).

[4] M. Jachura, M. Lipka, M. Jarzyna, and K. Banaszek, Opt. Express 25, 27475 (2017).

[5] M. Jachura, M. Jarzyna, M. Lipka, W. Wasilewski, and K. Banaszek, Phys. Rev. Lett. 120, 110502 (2018).

[6] M. Lipka, M. Jarzyna, and K. Banaszek, IEEE J. Sel. Areas Commun. 38, 496 (2020).

[7] V. Thiel, A. O. C. Davis, K. Sun, P. D'Ornellas, X.-M. Jin, and B. J. Smith, Opt. Express 28, 19315 (2020).

[8] R.-B. Jin, T. Gerrits, M. Fujiwara, R. Wakabayashi, T. Yamashita, S. Miki, H. Terai, R. Shimizu, M. Takeoka, and M. Sasaki, Opt. Express 23, 28836 (2015).

[9] V. Prakash, A. Sierant, and M. W. Mitchell, Phys. Rev. Lett. 127, 043601 (2021).

[10] N. Montaut, O. S. M. na Loaiza, T. J. Bartley, V. B. Verma, S. W. Nam, R. P. Mirin, C. Silberhorn, and T. Gerrits, Optica 5, 1418 (2018).

[11] T. Gerrits, F. Marsili, V. B. Verma, L. K. Shalm, M. Shaw, R. P. Mirin, and S. W. Nam, Phys. Rev. A 91, 013830 (2015).

[12] V. V. Orre, E. A. Goldschmidt, A. Deshpande, A. V. Gorshkov, V. Tamma, M. Hafezi, and S. Mittal, Phys. Rev. Lett. 123, 123603 (2019).

[13] A. M. Kaufman, B. J. Lester, C. M. Reynolds, M. L. Wall, M. Foss-Feig, K. R. A. Hazzard, A. M. Rey, and C. A. Regal, Science 345, 306 (2014).

[14] R. Lopes, A. Imanaliev, A. Aspect, M. Cheneau, D. Boiron, and C. I. Westbrook, Nature (London) 520, 66 (2015).

[15] K. Toyoda, R. Hiji, A. Noguchi, and S. Urabe, Nature (London) 527, 74 (2015).

[16] J. Li, M.-T. Zhou, B. Jing, X.-J. Wang, S.-J. Yang, X. Jiang, K. Mølmer, X.-H. Bao, and J.-W. Pan, Phys. Rev. Lett. 117, 180501 (2016).

[17] M. Parniak, M. Mazelanik, A. Leszczyński, M. Lipka, M. Dabrowski, and W. Wasilewski, Phys. Rev. Lett. 122, 063604 (2019).

[18] N. Uribe-Patarroyo, A. Fraine, D. S. Simon, O. Minaeva, and A. V. Sergienko, Phys. Rev. Lett. 110, 043601 (2013). 
[19] R. Fickler, R. Łapkiewicz, W. N. Plick, M. Krenn, C. Schaeff, S. Ramelow, and A. Zeilinger, Science 338, 640 (2012).

[20] J. Leach, B. Jack, J. Romero, A. K. Jha, A. M. Yao, S. Franke-Arnold, D. G. Ireland, R. W. Boyd, S. M. Barnett, and M. J. Padgett, Science 329, 662 (2010).

[21] M. Karpiński, M. Jachura, L. J. Wright, and B. J. Smith, Nat. Photonics 11, 53 (2017).

[22] M. Kues, C. Reimer, P. Roztocki, L. R. Cortés, S. Sciara, B. Wetzel, Y. Zhang, A. Cino, S. T. Chu, B. E. Little et al., Nature (London) 546, 622 (2017).

[23] T. Nitsche, S. De, S. Barkhofen, E. Meyer-Scott, J. Tiedau, J. Sperling, A. Gábris, I. Jex, and C. Silberhorn, Phys. Rev. Lett. 125, 213604 (2020).

[24] S. L. McCall and E. L. Hahn, Phys. Rev. Lett. 18, 908 (1967).

[25] M. D. Crisp, Phys. Rev. A 1, 1604 (1970).

[26] L. S. Costanzo, A. S. Coelho, D. Pellegrino, M. S. Mendes, L. Acioli, K. N. Cassemiro, D. Felinto, A. Zavatta, and M. Bellini, Phys. Rev. Lett. 116, 023602 (2016).

[27] D. Meshulach and Y. Silberberg, Nature (London) 396, 239 (1998).

[28] A. Devolder, P. Brumer, and T. V. Tscherbul, Phys. Rev. Lett. 126, 153403 (2021).

[29] A. J. A. Carvalho, R. S. N. Moreira, J. Ferraz, S. S. Vianna, L. H. Acioli, and D. Felinto, Phys. Rev. A 101, 053426 (2020).

[30] A. Marian, M. C. Stowe, J. R. Lawall, D. Felinto, and J. Ye, Science 306, 2063 (2004).

[31] C. Polycarpou, K. N. Cassemiro, G. Venturi, A. Zavatta, and M. Bellini, Phys. Rev. Lett. 109, 053602 (2012).

[32] See Supplemental Material at http://link.aps.org/ supplemental/10.1103/PhysRevLett.127.163601 for additional calculations and derivations, details on the role of photons' spectral correlations, phase reconstruction strategy, visibility estimates, comparison with homodyne tomography, and derivation of Fisher information for estimating the residual distinguishability, which includes Refs. [33-44].

[33] A. Nesmeyanov and R. Gary, Vapor Pressure of the Chemical Elements (Elsevier, New York, 1963).

[34] U. Kallmann, S. Brattke, and W. Hartmann, Phys. Rev. A 59, 814 (1999).

[35] P. Kolenderski, W. Wasilewski, and K. Banaszek, Phys. Rev. A 80, 013811 (2009).

[36] S. A. Karout, M. A. Gdeisat, D. R. Burton, and M. J. Lalor, Appl. Opt. 46, 730 (2007).

[37] D. J. Bone, H.-A. Bachor, and R. J. Sandeman, Appl. Opt. 25, 1653 (1986).

[38] S. M. Kay, Fundamentals of Statistical Signal Processing: Estimation Theory (PTR Prentice Hall, NJ, 1993).

[39] P. Sekatski, N. Sangouard, F. Bussières, C. Clausen, N. Gisin, and H. Zbinden, J. Phys. B 45, 124016 (2012).
[40] W. P. Schleich, Quantum Optics in Phase Space (WileyVCH, New York, 2001), ISBN 978-3-527-29435-0.

[41] J. C. Garrison and R. Y. Chiao, Quantum Optics (Oxford University Press, New York, 2008), ISBN 9780198508861.

[42] J. T. Henriksson, A. M. McDermott, and J.P. G. Bergmanson, Invest. Ophthalmol. Visual Sci. 50, 3648 (2009).

[43] H. Tang, X. Liu, S. Chen, X. Yu, Y. Luo, J. Wu, X. Wang, and L. Liu, IEEE Trans. Biomed. Eng. 66, 1803 (2019).

[44] S. L. Jacques, Phys. Med. Biol. 58, R37 (2013).

[45] R. Wallauer, M. Raths, K. Stallberg, L. Münster, D. Brandstetter, X. Yang, J. Güdde, P. Puschnig, S. Soubatch, C. Kumpf et al., Science 371, 1056 (2021).

[46] M. J. Rosker, M. Dantus, and A. H. Zewail, Science 241, 1200 (1988).

[47] M. Dantus, M. J. Rosker, and A. H. Zewail, J. Chem. Phys. 87, 2395 (1987).

[48] E. D. Potter, J. L. Herek, S. Pedersen, Q. Liu, and A. H. Zewail, Nature (London) 355, 66 (1992).

[49] P. Brumer and M. Shapiro, Annu. Rev. Phys. Chem. 43, 257 (1992).

[50] S. Mukamel, M. Freyberger, W. Schleich, M. Bellini, A. Zavatta, G. Leuchs, C. Silberhorn, R. W. Boyd, L. L. Sánchez-Soto, A. Stefanov et al., J. Phys. B 53, 072002 (2020).

[51] K. E. Dorfman, F. Schlawin, and S. Mukamel, Rev. Mod. Phys. 88, 045008 (2016).

[52] K.E. Dorfman, S. Asban, B. Gu, and S. Mukamel, Commun. Phys. 4, 49 (2021).

[53] T. S. Rose, M. J. Rosker, and A. H. Zewail, J. Chem. Phys. 88, 6672 (1988).

[54] B. L. Brown, A. J. Dicks, and I. A. Walmsley, Phys. Rev. Lett. 96, 173002 (2006).

[55] C. Meng, G. A. Brawley, J. S. Bennett, M. R. Vanner, and W. P. Bowen, Phys. Rev. Lett. 125, 043604 (2020).

[56] C. a. Klinteberg, A. Pifferi, S. Andersson-Engels, R. Cubeddu, and S. Svanberg, Appl. Opt. 44, 2213 (2005).

[57] Y. Hori, T. Yasui, and T. Araki, Opt. Rev. 13, 29 (2006).

[58] F. Wolfgramm, C. Vitelli, F. A. Beduini, N. Godbout, and M. W. Mitchell, Nat. Photonics 7, 28 (2013).

[59] M. Lipka and M. Parniak, Opt. Lett. 46, 3009 (2021).

[60] M. Lipka, M. Parniak, and W. Wasilewski, Appl. Phys. Lett. 112, 211105 (2018).

[61] R. Jozsa, J. Mod. Opt. 41, 2315 (1994).

[62] Z. Qin, A. S. Prasad, T. Brannan, A. MacRae, A. Lezama, and A. I. Lvovsky, Light Sci. Appl. 4, e298 (2015).

[63] C. Ferrie, Phys. Rev. Lett. 113, 190404 (2014).

[64] B. Brecht, D. V. Reddy, C. Silberhorn, and M. G. Raymer, Phys. Rev. X 5, 041017 (2015).

[65] J. M. Lukens and P. Lougovski, Optica 4, 8 (2017).

[66] A. Khodadad Kashi and M. Kues, Laser Photonics Rev. 15, 2000464 (2021). 